\title{
EL POSICIONAMIENTO ARGENTINO FRENTE A LOS PARADIGMAS TECNOECONOMICOS GLOBALES
}

\author{
Ignacio De Angelis*
}

enviado: septiembre 2016 - aceptado: noviembre 2016

\begin{abstract}
Resumen
El artículo busca problematizar los principales elementos asociados al cambio tecnológico que dieron forma a la inserción de Argentina en los distintos paradigmas tecnoeconómicos globales. En particular, se presenta una caracterización de los sucesivos modelos de desarrollo vigentes en el país desde 1930 y su posicionamiento internacional de acuerdo a la distribución de capacidades tecnológicas de la economía mundial.

Código JEL: F6, N10, O54.

Palabras clave: Desarrollo tecnológico, paradigmas tecnoeconómicos, Argentina.
\end{abstract}

\begin{abstract}
The objective of this article is to discuss the main elements associated with the technological change that gave shaped to the international insertion of Argentina into each global techno-economic paradigm. The paper submits a characterization of the successive development models in force in Argentina since 1930 and the international position according to the distribution of technological capabilities of the global economy.
\end{abstract}

JEL Code: F6, N10, O54.

Keywords: Technological development, techno-economic paradigm, Argentina.

* deangelisignacio@gmail.com Investigador CEIPIL/CIC - UNICEN - CONICET. Pinto 399 piso 3, Tandil. 


\section{INTRODUCCIÓN}

El objetivo del presente artículo es caracterizar las distintas etapas del posicionamiento argentino frente a cada paradigma tecnoeconómico global desde el siglo XX hasta la actualidad, haciendo hincapié en la formación de las capacidades tecnológicas de los distintos modelos de desarrollo que se suceden en Argentina a partir de 1930. Para ello, se prestará particular atención a la industria manufacturera y al sistema de innovación como ejes centrales en la adaptación y generación de tecnología.

A nivel doméstico la periodización del trabajo aborda la etapa correspondiente con el surgimiento de la estrategia de industrialización por sustitución de importaciones durante la primera mitad del siglo XX, su posterior desarticulación con la emergencia y crisis del modelo neoliberal, y la reconversión neodesarrollista de comienzos del siglo XXI. A nivel global, este período se corresponde con dos paradigmas tecnoeconómicos distintos que proyectan la distribución de capacidades científicas y tecnológicas del sistema internacional sin modificar la condición periférica de las pautas de inserción argentina.

La dinámica sistémica de la economía mundial presenta una morfología heterogénea en la distribución de beneficios del tablero tecnológico global, dando lugar a la sucesión de paradigmas. Los países centrales, de avanzado desarrollo industrial, dominan las posiciones de mercado de los productos de mayor valor agregado y nivel de tecnología. Por su parte, la inserción de los países periféricos está orientada a la especialización en recursos naturales y bienes de menor contenidos tecnológico. Sin embargo, las tecnologías son difundidas hacia todo el sistema, dado que en momentos de bajos rendimientos en las economías centrales con mercados saturados (símbolo del agotamiento del paradigma) los movimientos de capitales hacia la periferia buscan compensar la caída de los beneficios a partir de la transferencia y difusión - de carácter adaptativo- de tecnologías maduras junto con importantes movimientos financieros.

Esta difusión asimétrica responde tanto a los ciclos de rentabilidad de capital como a la dinámica propia del cambio tecnológico, exponiendo límites en la movilidad del conocimiento a partir de mecanismos de protección intelectual, generación de externalidades y la segmentaciones productivas que sostienen las actividades de $\mathrm{I}+\mathrm{D}$ en los países de origen y casas matrices dueñas de la tecnología. Asimismo, como en el caso de Argentina, las inversiones financiadas con ahorro externo suelen agudizar problemas estructurales conduciendo a crisis financieras y económicas (Schvarzer, 1996; Bresser - Pereira y Gala, 2008). 
De todas maneras, el carácter asimétrico en las relaciones entre países no asume una condición determinista de la dependencia, sino que reconoce la coexistencia de dinámicas internas y externas que definen las condiciones para el cambio tecnológico y el posicionamiento internacional.

A nivel interno existen dinámicas propias de la economía local y del sistema de innovación que ajustan las condiciones para la inserción comercial y tecnológica internacional. A nivel externo, cada paradigma ofrece una determinada configuración de elementos que dan forma al sistema internacional extrapolando dinámicas propias de la relación centro-periferia, principalmente respecto al direccionamiento de los flujos de tecnología durante los procesos de desarrollo e industrialización, reconfigurando las condiciones de dependencia tecnológica en los países periféricos. Sin embargo, por otro lado, cada paradigma tecnológico se estructura en torno a un conjunto de industrias y recursos claves que, de acuerdo a la disponibilidad y las capacidades propias de cada país, condicionan el posicionamiento tanto de los países centrales como periféricos.

Frente a este escenario adquieren relevancia las políticas tendientes a desarrollar los sistemas propios de ciencia, tecnología e innovación, colocando el conocimiento, el aprendizaje y la interacción entre actores, como base para la formación de capacidades tecnológicas. En particular, como síntesis de los esfuerzos desplegados, interesa analizar la triangulación de relaciones entre el gobierno, el sector productivo y el académico (o científico) como elemento dinamizador, entendiendo que su interacción permite incorporar la ciencia y la tecnológica en las estrategias de desarrollo socioeconómico desde una perspectiva que permite avanzar hacia una mayor autonomía en los países periféricos (Sábato y Botana, 1970)1.

Con todo, la argumentación del artículo sostiene que el cambio tecnológico y la acumulación de conocimiento tienen lugar de forma sistémica y contextualizada, es decir, en constante interacción con actores e instituciones, adquiriendo particularidades propias al marco social, económico, político y cultural donde se inscribe.

La hipótesis principal que guía el trabajo es que existen dinámicas inherentes a la sucesión de los distintos paradigmas tecnoeconómicos del sistema internacional que incidieron en la formación de las capacidades tecnológicas argentinas,

1 Los aportes de Jorge Sábato dieron lugar al modelo analítico conocido como Triangulo de Sábato, que posteriormente derivaría en una reformulación desde los países centrales como el enfoque de la Triple Hélice, abandonando la pretensión de autonomía de la formulación original (De Angelis, 2015). 
impulsando y limitando transformaciones en la estructura productiva y en las formas de regulación de las estrategias de desarrollo adoptadas en el país, y que en su acontecer fueron cristalizando la condición periférica y la dependencia tecnológica.

En la primera parte del trabajo se presenta la conceptualización que enmarca la discusión en torno a los paradigmas tecneconómicos y la centralidad de los proceso de formación de capacidades tecnológicas para el posicionamiento de los países en la economía internacional. En segundo lugar, el artículo avanza en el análisis del caso argentino frente a los dos últimos paradigmas. Por último, se presentan algunas reflexiones en torno a la formación de las capacidades tecnológicas.

\section{PARADIGMAS TECNOECONÓMICOS Y DESARROLLO}

El concepto sistémico de paradigma tecnoeconómico (PTE) responde a una visión holística del conjunto de las relaciones tecnológicas en todos los planos. Un PTE puede ser definido como el conjunto de principios tecnológicos y organizacionales difundidos y generalizados que se expresan como sentido común "para la organización de cualquier actividad y la reestructuración de cualquier institución", y que representan la "forma más efectiva de aplicar la revolución tecnológica y de usarla para modernizary rejuvenecer el resto de la economía" (Pérez, 2004, p. 41). De este modo, por su alcance, un PTE condiciona el comportamiento futuro de la economía en su conjunto, afectando directamente o indirectamente a casi todas las ramas y formas de producción (Freeman y Pérez, 2003).

La idea central que subsiste en la relación entre el desarrollo económico y los paradigmas tecnoeconómicos es que las distintas revoluciones tecnológicas, a partir de una tecnología matriz, impulsaron el desarrollo de nuevos sectores productivos enteros -desde la máquina de vapor hasta la biotecnología, pasando por el ferrocarril, el telégrafo intercontinental y la aeronáutica- con consecuencias en los régimen de producción y consumo de todos los rincones geográficos del planeta. Como consecuencia, el mapa internacional centro-periferia fue variando de acuerdo a las condiciones de inserción de los países en cada paradigma, fundamentalmente determinadas por las capacidades tecnológicas (de generación y aprendizaje) asociadas a dicho PTE, como también por la disponibilidad de recursos fundamentales y las condiciones de movilidad y difusión pautadas desde los países centrales.

Pérez y Freeman (2003) sostienen que los cambios en el paradigma marcan una crisis estructural y un ajuste, que requiere, como respuesta, un cambio social e institucional de adaptación de carácter sistémico. Una vez que se produce 
la selección de las nuevas tecnologías y su adecuación social (necesariamente conflictivas $)^{2}$, devienen períodos de estabilidad de la inversión en el largo plazo por dos o tres décadas, dando lugar a una fase ascendente en la formación las ondas larga de desarrollo a nivel internacional ${ }^{3}$. Por su parte, durante las fases recesivas (donde comienza la difusión hacia la periferia), se puede identificar saturación de mercados sectoriales centrales en un marco de inestabilidad de la economía internacional, con políticas monetarias restrictivas, incertidumbre tecnológica, proteccionismo y caída de la actividad.

A partir de allí, los paradigmas se difunden y suceden. Los nuevos tienen origen dentro del vigente, dando lugar a transformaciones en las formas de producción y de regulación nacional en cada país, y a los cambios en el sistema internacional con el surgimiento de nuevos países líderes y alteraciones relativas en la división internacional del trabajo.

Siguiendo a Pérez (2009), es posible identificar grandes oleadas de desarrollo de la economía capitalista internacional asociadas a las revoluciones científicas y tecnológicas que tuvieron lugar sucesivamente a partir de la primera revolución industrial. De este modo, se establece una periodización de cinco etapas históricas donde cada una corresponde a un paradigma tecneconómico: a) desde la primera revolución industrial con centro en Inglaterra desde 1770 y caracterizada como de mecanización temprana; b) la segunda desde 1830 marcada por el desarrollo del vapor y ferrocarriles, tiene lugar en Inglaterra y rápidamente se extiende a Europa y Estados Unidos; c) la tercera desde 1880 a partir del desarrollo y difusión del acero, electricidad e ingeniería pesada; d) cuarta etapa que comienza hacia 1910/1930 centrada en el petróleo y automóvil; y e) la más reciente desde 1970/90 caracterizada por el desarrollo de las tecnologías información y comunicación.

Cada uno de estos paradigmas permite visibilizar la distribución de capacidades tecnológicas como elemento para explicar las relaciones económicas internacionales modernas. En particular, adquiere relevancia la problematización de la inserción de Argentina en los distintos paradigmas tecnoeconómicos,

2 Proceso asociado a la idea de "destrucción creativa" propuesto por Schumpeter (1939).

3 El impacto de la tecnología en la formación de ciclos fue ampliamente abarcado por la teoría económica. La periodización para la formación de los PTE propuesta por Pérez (a partir de 2009), toma distancia de la caracterización temporal de los ciclos de onda larga por presentar irregularidades en la sustanciación empírica. Se sigue que cada revolución tecnológica marca el inicio de una "gran oleada de desarrollo" (2009: 20) y de transformación de la economía global, que puede extenderse más allá de la temporalidad propuesta en los ciclos de onda larga (ciclos de Kondriatiev) y su asociación a las revoluciones industriales (Schumpeter, 1939). 
interpretando el proceso de formación de capacidades como una de las variables analíticas claves para el estudio de las condiciones y determinantes del posicionamiento sistémico y las estrategias de desarrollo adoptadas.

Para este trabajo en particular, y luego de un sucinto repaso de lo acontecido frente a los primeros paradigmas, se desarrolla un análisis del posicionamiento argentino durante el cuarto y quinto PTE, exponiendo la caracterización de las principales variables analíticas (Tablas 1 y 2), entre las cuales se destacan: sectores industriales dinámicos; grandes inversiones en infraestructura; innovaciones centrales; principales fuentes de energía; cambios en el sistema internacional a partir del surgimiento de nuevos países líderes; y formas de regulación nacional que adoptan los distintos países.

\section{EL POSCIONAMIENTO ARGENTINO FRENTE A LA SUCESIÓN DE PARADIGMAS}

Durante la vigencia del primer PTE, la posición marginal de los territorios coloniales dentro de la economía mundial estuvo determinada por la situación de atraso en la que se instituyó el régimen político dominante (Jaguaribe, 1971). Luego de la Revolución Industrial, la región se especializó en la producción de materias primas para alimentar la creciente expansión de los países centrales. Durante el segundo paradigma (1830-1880) tuvo lugar el impulso a la inserción comercial argentina a partir de los mayores volúmenes de producción, resultado de la organización estatal y la explotación de la pampa húmeda. Por su parte, a partir de 1880, y en vigencia del tercer PTE, se produjo el auge del modelo agroexportador junto con la conformación de las primeras industrias de baja complejidad. Sin embargo, en un marco de importantes modificaciones técnicas en las prácticas agropecuarias a nivel global, el escaso desarrollo de capacidades tecnológicas para adaptar las nuevas técnicas de producción de insumos y equipamiento, forzó la dependencia de importaciones a partir de la disponibilidad de divisas que brinda la pampa húmeda ${ }^{4}$.

Respecto al perfil de las manufacturas locales durante los primeros paradigmas, existen una serie de elementos que explican su atraso relativo (Vitelli, 2012). En primer lugar, estuvieron orientadas mayormente a la cobertura de necesidades básicas; en segundo lugar, constituían una expresión trunca en cuanto a la falta de

4 Frente a las nuevas tecnologías globales (maquinaria agrícola, energía y genética vegetal en agroquímicos) existió por parte de ciertos actores locales una subestimación de las innovaciones junto con el agotamiento de las tierras disponibles para la explotación agropecuaria (Vitelli, 2012; Schvarzer, 1996). 
desarrollo de los eslabonamientos con la metalurgia, dada la inexistencia de producción minería y de combustibles en el país; en tercer lugar, el tamaño de las unidades y la falta de infraestructura impidió la formación de escalas productivas para impulsar el desarrollo tecnológico; y en cuarto lugar, no se generó una asociación de intereses entre el Estado y los actores industriales. Como resultado, no existieron condiciones para la formación de capacidades tecnológicas que permitan sostener un sistema productivo de acuerdo al paradigma tecnoeconómico vigente.

II. 1. Argentina frente al cuarto paradigma: industria sustitutiva y modelo fordista periférico

A nivel global, el cuarto paradigma tecnoeconómico se caracterizó por el auge del régimen de acumulación fordista keynesiano y su posterior crisis asociada a la rigidez del modelo en sus formas de regulación y acumulación (Tabla 1). A nivel interno, coincidió con la crisis del modelo agroexportador, signada por los cambios en el contexto internacional y límites de la capacidad productiva interna.

La adopción del modelo de desarrollo de industrialización por sustitución de importaciones (ISI) tuvo lugar frente a la crisis de 1930 y la caída de los precios de los productos exportables, junto con la interrupción de los flujos comerciales y tecnológicos globales en el marco de las grandes guerras 5 .

En el plano global, los cambios y la disputa por la hegemonía luego de la crisis de 1929 y de la segunda guerra posicionaron a Estados unidos como nuevo líder económico y financiero a nivel global, con una fuerte presencia en América Latina en general y en Argentina en particular.

A nivel local, la evolución política hacia un nuevo modelo de desarrollo, su inserción comercial y la relación con los socios estratégicos, pusieron en juego las alianzas forjadas durante el modelo anterior. Como resultado de tensiones particulares sobre la economía doméstica en un marco de sucesivos ajustes, se generó la base de una nueva coalición policlasista entre parte de la burguesía urbana (industriales no concentrados) y el sector popular organizado (O'donnell, 1977).

Durante la guerra, producto del sostenimiento de las exportaciones agropecuarias, crecieron los saldos positivos en libras con Gran Bretaña, al mismo tiempo

5 Siguiendo a Schvarzer (1996), la nueva estrategia fue aceptada por las elites como respuesta necesariamente temporal a la crisis del comercio internacional. 
Tabla 1. IV Paradigma. 1910/1930 - La era del automóvil y el uso difundido del petróleo

\begin{tabular}{|c|c|}
\hline Ramas Líderes & $\begin{array}{l}\text { Automóviles, tractores, armamento pesado, aviones, bienes } \\
\text { de consumo durable, petroquímica y material sintético. }\end{array}$ \\
\hline $\begin{array}{l}\text { Infraestructura y matriz } \\
\text { energética }\end{array}$ & $\begin{array}{l}\text { Rutas y aeropuertos. Electricidad y telecomunicaciones } \\
\text { analógicas a nivel global. Grandes escalas para a produc- } \\
\text { ción. Redes de oleoductos. Auge hidrocarburos. }\end{array}$ \\
\hline Industrias Clave & Energía, derivada principalmente del petróleo, y petroquímica. \\
\hline Principales Innovaciones & $\begin{array}{l}\text { Mercado de masas. Integración horizontal. Estandariza- } \\
\text { ción de la producción y el consumo. Motor de combustión } \\
\text { interna. Auge sintético. Comunicación inalámbrica }\end{array}$ \\
\hline $\begin{array}{l}\text { Limitación del paradigma } \\
\text { anterior }\end{array}$ & $\begin{array}{l}\text { Problemas de escala y estandarización de la producción } \\
\text { para el sostenimiento de los cada vez mayores niveles de } \\
\text { consumo. }\end{array}$ \\
\hline $\begin{array}{l}\text { Sector en Crecimiento } \\
\text { (impulsan } \mathrm{V}^{\circ} \mathrm{PTE} \text { ) }\end{array}$ & $\begin{array}{l}\text { Computadoras, radares, nuevas maquinarías de produc- } \\
\text { ción, industria farmacéutica, armas nucleares, desarrollo } \\
\text { de software y microelectrónica. }\end{array}$ \\
\hline Países líderes & $\begin{array}{l}\text { Estados Unidos y Alemania. Difusión hacia Europa. Auge } \\
\text { multilateralidad en las Relaciones Internacionales: ONU, } \\
\text { OTAN, G7, FMI, BIRF, GATT- OMC. }\end{array}$ \\
\hline Regímenes de regulación & $\begin{array}{l}\text { Auge del modelo fordista - keynesiano. Estado de } \\
\text { Bienestar. Movimiento de la liquidez mundial de manos } \\
\text { privadas a manos públicas. Crisis y desarticulación. }\end{array}$ \\
\hline Sistemas de Innovación & $\begin{array}{l}\text { Expansión de los departamentos de I+D. Asociación } \\
\text { Innovación - consumo. Impulsos estatales a la I+D en } \\
\text { el campo militar dando forma a la big science. Modelos } \\
\text { centrados en la oferta del conocimiento. }\end{array}$ \\
\hline Argentina & $\begin{array}{l}\text { Modelo ISI y desarrollo gran infraestructura. Ciclos de } \\
\text { Stop and go. 1960-1970 se produce la incorporación del } \\
\text { nuevo paquete tecnológico al agro. }\end{array}$ \\
\hline SNI (Argentina) & $\begin{array}{l}\text { Institucionalización de la ciencia y la tecnología. Base de } \\
\text { actual Sistema Nacional de Innovación (SNI). Primeros } \\
\text { laboratorios I+D en el sector productivo. }\end{array}$ \\
\hline
\end{tabular}

Fuente: Elaboración propia en base a Freeman y Pérez (2003), Vitelli (2012), Schvarzer (1996) y De Angelis (2015). 
que los productos industriales británicos redujeran su presencia en el mercado internacional, permitiendo que Argentina experimente un auge de las exportaciones industriales -y la formación de capacidades tecnológicas asociadas- impulsado por el comercio con América Latina ${ }^{6}$.

La participación de los productos industriales en el total de las exportaciones pasó de 1,4\% entre 1936 a un máximo de 19,4\% en 1943. Con el fin de la guerra, las exportaciones industriales caen producto del retorno a los mercados de las potencias centrales. Hacia 1947 el porcentaje se reduzca a 5,5\%, compensando parcialmente la cuenta corriente por las ventas de productos primarios (Llach, 1984).

Si bien existió un importante acercamiento hacia la región, los volúmenes comerciales registrados fueron relativamente bajos. Luego de la guerra, y hasta la década de 1970, la industria siguió desarrollándose pero sin el impulso exportador de los años previos, por lo que se consolida el perfil mercado internista.

Por otro lado, como corolario de la estrategia industrialista sobre una base dependiente de desarrollo tecnológico crecieron las importaciones provenientes de Estados Unidos. Los capitales estadounidenses que habían ingresado con fuerza desde finales del período anterior, se constituyeron como un fuerte factor de presión para direccionar el aprovisionamiento de importaciones estratégicas, como combustibles, insumos intermedios y bienes de capital (Rapoport, 2000).

El principal desafío tecneconómico se cristalizó en la capacidad para sostener un equilibrio en la balanza de pagos capaz de resistir las importaciones estratégicas que requería el proceso de industrialización y que obligaban a sostener e incrementar la venta externa de productos agropecuarios y manufacturas a cambio de las divisas necesarias para evitar la restricción externa. En este marco, la necesidad de divisas permeo la llegada de capitales extranjeros orientadas a la industria y los servicios.

Para la década del 1960 era evidente que la apertura a las IED no aportó soluciones a la restricción externa, sino que fue generando una base estructural de enclaves transnacionales concentrados que, posteriormente, con el neoliberalismo y el proceso de "privatización periférica" de la empresas concesionarias de servicios públicos, terminó por desregular las contrataciones con proveedores locales, apuntalando la desarticulación productiva en favor de la participación en cadena globales de valor y descentralizando el proceso de valorización tecnológica (Katz, 1972; Schvarzer, 1996).

6 Las exportaciones Argentinas hacia la región se incrementaron de $20 \%$ a inicios de la guerra hasta el 47,3\% para los años 1943-1945 (Llach, 1984). 
Frente a estos límites adquirió centralidad el proceso de cambio tecnológico como elemento dinámico para avanzar hacia mayores márgenes de autonomía vía generación de capacidades productivas que permitan reducir el peso de las importaciones. En efecto, resulta clave el desarrollo del sistema de innovación durante el período, dado que allí surgieron las instituciones inaugurales del actual complejo científico y tecnológico.

Durante la década del cincuenta, se fue constituyendo el eje central del sistema de innovación con la creación del Instituto Nacional de Tecnología Agropecuaria (INTA), el Instituto Nacional de Tecnología Industrial (INTI), y la reconversión en 1958 del CONICYT en el Consejo Nacional de Investigaciones Científicas y Técnicas (CONICET). Asimismo, desde mediados de la década del cuarenta y hasta fines de los cincuenta fueron creados otras instituciones y organismos específicos que dieron densidad al sistema.

En este marco, la pauta signada por los desafíos tecnológicos de la estructura productiva local se expresó en la importancia que evidenciaron en la tarea de $\mathrm{I}+\mathrm{D}$ y en el fortalecimiento e integración de proveedores locales, las grandes empresas estatales como Obras Sanitarias de la Nación, Ferrocarriles Argentinos y Yacimientos Petrolíferos Fiscales (YPF). Del mismo modo, en este campo y en el relacionamiento del poder militar en la esfera productiva tuvo un rol fundamental el Ministerio de Defensa (López, 2002).

La información empírica señala la existencia de un proceso de formación de capacidades tecnológicas dentro de la industria, tanto por la dinámica de producción interna ampliada como también, y en mayor medida, como respuesta al proceso de aprendizaje e incorporación de tecnología. El analizar la evolución de las exportaciones según su densidad tecnológica, hacia los últimos años del modelo es posible advertir señales de un proceso de escalonamiento en la composición de los bienes. Para 1962, según datos del Centro de Estudios Internacionales del Ministerio de Economía, el $80 \%$ de las exportaciones estaba compuesto por productos primarios (fundamentalmente trigo, carne, maíz y lana) y el $17.8 \%$ por manufacturas basadas en recursos naturales. Mientras que en 1974, las exportaciones de productos primarios se redujeron al $54.4 \%$ y las exportaciones industriales basadas en recursos naturales aumentaron al $24.3 \%$.

De esta manera, si bien el volumen de las exportaciones condujo los distintos ciclos del crecimiento económico que derivó en la desarticulación política del modelo industrialista, lo cierto es que existió una reorientación de las capacidades de inserción en términos del paradigma tecnoeconómico internacional. No sólo 
fueron impulsadas aquellas industrias motorizadas por el mercado interno, sino que se produjo una importante difusión de sectores asociados a la industria pesada y a las actividades líderes.

No obstante, la dependencia de componentes básicos de producción para sostener el modelo industrialista fue generando una brecha en las capacidades competitivas internacionales que sostuvo la viabilidad de las empresas locales solo a partir del sistema de protección y la orientación hacia el mercado interno. Asimismo, es posible advertir que la formación de capacidades tecnológicas reflejan el desacople respecto a la dinámica de la innovación a nivel global, que ya presentaba evidencias de la existencia de las nuevas tecnologías transformadoras que impulsaban el próximo PTE (Tablas 1 y 2).

A comienzos de la década de 1970 los esfuerzos industrializadores se vieron interrumpidos por una nueva crisis internacional y el impacto en los precios relativos y en los términos de intercambio. Al mismo tiempo, desde la década anterior y producto de la incorporación local de la denominada "revolución verde", se fueron recuperando posiciones relativas dentro del mapa global de productores agropecuarios $^{7}$. En suma, el aumento de los precios primarios y el auge de los petrodólares ofrecieron una salida transitoria a los problemas de balance de pagos agudizando las limitaciones estructurales.

Con todo, la interrupción del proceso de industrialización socavó la emergencia de una base productiva consciente de la formación de capacidades tecnológicas como elementos para enfrentar las condiciones de inserción internacional, retomando, durante la fase de despliegue del quinto paradigma, una visión ortodoxa y dependiente de la producción y movilidad del conocimiento y la tecnología.

II. 2. Argentina frente al paradigma actual: divergencias para la consolidación del SNI

Desde la década del setenta se fueron generando importantes cambios asociados al surgimiento del quinto PTE impulsado por la electrónica y las nuevas tecnologías de la información y la comunicación (Tabla 2). Hasta este período, las distintas revoluciones que estimularon la formación de paradigmas estuvieron

7 La biología y la genética impulsaron aumentos en los rindes, junto con el desarrollo de nuevas técnicas reproductivas y avances en la química agropecuaria y la industria farmacéutica animal. Asimismo, estos avances estuvieron acompañados por el desarrollo de nueva maquinaria de gran porte y mayor flexibilidad (Vitelli, 2012). 
asociadas mayormente -al menos en sus inicios- con transformaciones en el uso de la energía. Por su parte, la revolución científica y tecnológica inaugural del quinto paradigma tuvo lugar en el ámbito de la información y la difusión del conocimiento, atravesando el conjunto de las actividades humanas e impulsando cambios sistemáticos a nivel productivo, organizativo, institucional y cultural.

El cambio en los modos de organización de las empresas y la redefinición de los espacios y las pautas de producción y consumo, arrojó un nuevo escenario de competitividad internacional basado en el conocimiento, dando origen a un nuevo régimen de acumulación capitalista posfordista sostenido en un esquema de producción flexible y deslocalizado.

En este marco de transformaciones sistémicas, a nivel local la dictadura militar avanzó en la desarticulación de la estrategia sustitutiva, dando paso a un modelo de desarrollo neoliberal, que continuó, con matices, en los posteriores gobiernos democráticos y alcanzó su máxima expresión en la década del noventa durante la vigencia de la ley de convertibilidad cambiaria.

Las reformas estructurales neoliberales impulsaron una transformación productiva ampliando la heterogeneidad (entre sectores e intra sectorial). Se produjo la especialización en industrias basadas en recursos naturales y commodities industriales (de bajo y medio contenido tecnológico), como también el auge de la industria automotriz. Complementariamente, se impulsó la modernización en infraestructura, energía y telecocomunicaciones frente a una nueva etapa de la globalización y una creciente importancia de las firmas transnacionales y los grandes grupos económicos locales (apuntalando la extranjerización y concentración de las estructuras económicas). Como resultado, se produjo el desplazamiento relativo de sectores sensibles para el desarrollo, como son las manufacturas intensivas en mano de obra y los sectores de mayor complejidad tecnológica, generando la "llegada masiva de importaciones" y la "desaparición de capacidades tecnológicas (Katz, 2000, p. 27 y 48)

Paralelamente, el impacto de la "revolución verde" dotó al sector agropecuario de un nuevo dinamismo, estrechando los vínculos con la innovación agruparía generada por firmas trasnacionales. Como consecuencia, desde la década de 1970 se introdujeron nuevos cultivos y se extendieron las zonas productivas más allá los espacios tradicionales, dando inicio a un proceso de transformación en la fisionomía local que culminó con la incorporación del paquete tecnológico sojero en la década de 1990, y que domina hasta la actualidad la producción del agro argentino y las industrias asociadas (Vitelli, 2012). 
Tabla 2: V Paradigma. 1970 - La era de la información y el conocimiento

\begin{tabular}{|c|c|}
\hline Ramas Líderes & $\begin{array}{l}\text { Computadoras, bienes electrónicos de capital, telecomunicaciones, soft- } \\
\text { ware, fibra óptica, robótica, satélites, comunicación digital, entre otros. }\end{array}$ \\
\hline $\begin{array}{l}\text { Infraestructura y } \\
\text { matriz energética }\end{array}$ & $\begin{array}{l}\text { Transporte físico y digital de alta velocidad. Mundialización de las } \\
\text { comunicaciones. Crisis de la matriz energética en torno a combus- } \\
\text { tibles fósiles. }\end{array}$ \\
\hline Industrias Clave & $\begin{array}{l}\text { Conocimiento e información como factores centrales de la produc- } \\
\text { ción y fuente de ventajas competitivas. Microelectrónica. }\end{array}$ \\
\hline $\begin{array}{l}\text { Principales } \\
\text { Innovaciones }\end{array}$ & $\begin{array}{l}\text { TICs. Nuevos límites del conocimiento. Uso intensivo de las nuevas } \\
\text { tecnologías. Especialización, diseño y marketing. Cadenas globales de } \\
\text { valor. Estructuras y sociedad en red. }\end{array}$ \\
\hline $\begin{array}{l}\text { Limitación del } \\
\text { paradigma anterior }\end{array}$ & $\begin{array}{l}\text { Rigidez de producción. El nuevo paradigma ofrece solución de flexibi- } \\
\text { lidad en tiempos y formas. Ensamblado, precisión y control de todo el } \\
\text { proceso productivo a partir de la incorporación de sistemas electrónicos }\end{array}$ \\
\hline $\begin{array}{c}\text { Sector en } \\
\text { Crecimiento } \\
\text { (impulsan VIo PTE) }\end{array}$ & $\begin{array}{l}\text { Biotecnología, nanotecnología e ingeniería robótica. Aplicación } \\
\text { de las TIC a la producción sustentable. Hiperconectividad. Código } \\
\text { abierto: comienza a percibirse que la protección como barrera al } \\
\text { desarrollo. Almacenamiento de energías renovables. }\end{array}$ \\
\hline Países líderes & $\begin{array}{l}\text { Comienzo y fin de la unipolaridad. Estados Unidos y Alemania. Di- } \\
\text { fundiéndose hacia Europa y luego Asia. Ascenso de China y disputa } \\
\text { de hegemonía. Apertura India. }\end{array}$ \\
\hline $\begin{array}{l}\text { Regímenes de } \\
\text { regulación }\end{array}$ & $\begin{array}{l}\text { Desarticulación del Estado de Bienestar. El mercado regulador. El Es- } \\
\text { tado desarrolla infraestructura y servicios básicos (y de seguridad). } \\
\text { Financierización e inestabilidad. Relocalización de la producción a } \\
\text { partir de las diferencias internacionales. Revalorización de lo local. }\end{array}$ \\
\hline $\begin{array}{l}\text { Sistemas de } \\
\text { Innovación }\end{array}$ & $\begin{array}{l}\text { SNI modernos (articulación virtuosa público - privada). Auge de la } \\
\text { innovación en la búsqueda de generación de ventajas competitivas } \\
\text { dinámicas. Mundialización de los regímenes de propiedad intelectual. }\end{array}$ \\
\hline Argentina & $\begin{array}{l}\text { Abandono ISI. Modelo de desarrollo neoliberal y crisis. Nuevos cul- } \\
\text { tivos y la extensión de tierras más allá de la pampa húmeda. Este } \\
\text { proceso se profundizó con la incorporación paquete soja. }\end{array}$ \\
\hline SIN (Argentina) & $\begin{array}{l}\text { Visión ortodoxa cambio tecnológico. Fin esfuerzos domésticos en } \\
\text { ciencia y tecnología. Desindustrialización y pérdida de capacidades. } \\
\text { Formalización y divergencia durante convertibilidad. Crisis neolibe- } \\
\text { ral, modelo neodesarrollista y nueva política científico tecnológica }\end{array}$ \\
\hline
\end{tabular}

Fuente: Elaboración propia en base a Freeman y Pérez (2003), Pérez (2009), Vitelli (2012) y De Angelis (2015). 
En este orden de análisis, Nochteff (2002) sostiene que la virtual ausencia presupuestaria de la política científica y tecnológica en Argentina durante el período neoliberal responde principalmente a la insuficiente demanda de innovaciones de los principales agentes económicos con mayor capacidad de influir en las decisiones políticas. Estos agentes obtuvieron beneficios extraordinarios a partir de un conjunto de "ventajas monopólicas no basadas en la innovación" (Nochteff, 2002, p558), y por lo tanto, no necesitaron del desarrollo de un sistema de innovación, ni de la formación de recursos humanos calificados para la obtención de ventajas competitivas dinámicas, sino, por el contrario, que basaron su obtención de beneficios sobre la disponibilidad de recursos naturales y en ventajas artificiales otorgadas a partir de subsidios, esquemas preferenciales, protecciones, concentración de mercados y libertades para fijar precios. Por su parte, las innovaciones asociados al sector agropecuario fueron mayormente incorporadas como paquetes tecnológicos generados exógenamente.

La extranjerización en los sectores más dinámicos (alimentos, metalurgia, industria química, petrolera e industria automotriz) condicionó la incorporación del cambio tecnológico, en tanto integran cadenas globales de valor, articulando sus funciones de producción con proveedores extranjeros y concentrando sus principales inversiones en $\mathrm{I}+\mathrm{D}$ fuera del país. Asimismo, mediante la remisión de utilidades y las compras e insumos y bienes de producción en el extranjero, generan problemas en la disponibilidad de divisas. Estas condiciones estructurales trascendieron la ruptura neoliberal que significó la crisis de la convertibilidad, profundizándose durante la vigencia del modelo neodesarrollista en los últimos años ${ }^{8}$.

En el campo científico y tecnológico, en un contexto de vulnerabilidad y crisis económicas recurrentes, la actividad tuvo un lugar marginal. El gobierno militar dio impulso a la investigación en temas nucleares y espaciales, pero lo hizo en un contexto de represión, intervención, desarticulación y aislamiento de las instituciones científicas, tecnológicas y de las universidades (Albornoz y Gordon, 2011). Por su parte, el gobierno de Alfonsín (1983-1989) recibió como herencia un país en crisis, endeudado, con una profunda crisis fiscal y caída de la inversión, situación que se agudizó con la hiperinflación y licuó los recursos de las universidades y las principales instituciones, agudizando su aislamiento (Nun, 1995).

8 El neodesarrollismo refiere a las nuevas estrategias (siglo XXI) de acumulación centradas en el crecimiento del sector industrial en un contexto globalizado (diferencia sustancial con el desarro1lismo, que impulsó la industrialización en un marco de protección) (Porta y Bianco, 2004; Curia, 2011). En Argentina, este modelo representó una continuidad negativa del orden neoliberal profundizando los elementos estructurales descriptos (Féliz, 2011). 
Durante los años noventa, el desarrollo científico y tecnológico siguió teniendo un lugar marginal que coincidió, sin embargo, con un conjunto de iniciativas formales en torno al auge internacional de los modelos de planificación basados en la innovación ${ }^{9}$.

Luego de la crisis de la convertibilidad, y a raíz de la emergencia de un nuevo modelo de desarrollo, se impulsaron una serie de cambios en referencia al marco legislativo, organizacional y presupuestario del sistema científico y tecnológico nacional ${ }^{10}$. Entre las iniciativas más importantes, y en línea con las trasformaciones del nuevo paradigma tecnoeconómico global y la inserción internacional de las capacidades locales, se encuentran la sanción de Ley de Promoción de la Industria del Software (que permitió un Importante desarrollo del software y servicios asociados), la creación de la Fundación Argentina de Nanotecnología y la Ley de Promoción de la Biotecnología Moderna. Posteriormente, durante el año 2007, tuvo lugar la creación del Ministerio de Ciencia, Tecnología e Innovación Productiva. Sin embargo, estos cambios expresaron un conjunto de limitantes estructurales en su relacionamiento con el sistema productivo.

$\mathrm{Al}$ analizar el posicionamiento tecnoeconómico mediante la estructura de importaciones y exportaciones de acuerdo al contenido tecnológico de los bienes en los últimos años, es posible advertir que si bien durante la pos convertibilidad existió un mayor crecimiento de las exportaciones de productos industriales, no existió un impacto diferencial en la escala de contenido tecnológico de los productos y se produjo la consolidación del perfil exportador en torno a los productos de bajo contenido tecnológico relacionados al procesamiento de recursos naturales.

En promedio, según datos del Banco Interamericano de Desarrollo, durante el período $2006-2015$, el $45 \%$ de las exportaciones argentinas fueron productos

9 Se produjo la formalización SNI pero en un marco de divergencia entre políticas implícitas y explícitas en la materia (De Angelis, 2015). El nacimiento formal puede señalarse en 1996 con la creación del GACTEC, la ANPCYT y luego la sanción de la Ley 25.467/2001, marco del SIN y de cumplimiento efectivo en la siguiente etapa.

10 El aumento presupuestario y la revitalización institucional se reflejaron en la evolución de los principales indicadores y en la formación de capacidades a nivel organizacional. Siguiendo los datos de la Red de Indicadores de Ciencia y Tecnología (RICYT), el gasto en actividades científicas y tecnológicas e I+D como porcentaje del PBI, registró máximos históricos en 2011 con un valor de $0,66 \%$. Se registró un crecimiento de los recursos humanos dedicados a la actividad, con un total de 4.76 investigadores cada 1000 personas de la PEA, lo cual es el máximo nivel alcanzado por el país aunque con salarios más bajos que los principales países de la región. Asimismo, se multiplicó la inversión en infraestructura. 
primarios, el $25 \%$ productos de medio y alto contenido tecnológico, y el resto manufacturas basadas en recursos naturales o de bajo contenido tecnológico. Por su parte, el análisis de las importaciones señala la preeminencia de los bienes industriales de media alta tecnología con un $63 \%$ del total ${ }^{11}$, un $28 \%$ de manufacturas basadas en recursos naturales o de bajo contenido tecnológico (impulsadas por las compras energéticas) y un $9 \%$ de productos primarios.

Asimismo, en un contexto de reactivación de la producción industrial, el análisis de la inversión y la formación de capital muestran un crecimiento acelerado de las compras de máquinas y equipos de origen extranjero, que alcanzaron un máximo en su participación del 65\% en 2011 respecto al total de inversiones en equipos de producción (De Angelis, 2015). Estas inversiones en bienes de capital no se encuentran asociados a la producción local en los nuevos sectores dinámicos del PTE, sino a los enclaves industriales consolidados durante el neoliberalismo, que desde una perspectiva científica y tecnológica constituyen sectores marginales y en declinación.

En este orden de análisis, el auge global de los productos primarios a comienzos del siglo XXI y los límites en la trasformación del marco de regulación local para las actividades primarias y extractivas, resultaron en un mantenimiento del esquema de distribución en la participación sectorial en la estructura del producto nacional, reproduciendo las pautas estructurales de inserción periférica aunque bajo evidencias de desarrollo de capacidades productivas, tecnológicas y de una nueva dinámica institucional.

De este modo, es posible señalar la reproducción de las pautas del posicionamiento tecnoeconómico periférico de la economía argentina durante el quinto paradigma en un momento donde comienzan a evidenciarse señales de agotamiento y emergencia de un nuevo PTE a nivel global. Es decir, que más allá de la formación de capacidades organizacionales y productivas en los últimos años, en el campo científico y tecnológico continúan primando condiciones estructurales del régimen de producción (concentración, primarización, y transnacionalización) que agudizan la baja demanda y la débil vinculación respecto a la oferta de conocimiento y los sectores más dinámicos del paradigma actual.

11 El trabajo de Bekerman (2015) muestra empíricamente que durante el período 2003-2013 se alimentó la dinámica de formación de la restricción externa vía alta elasticidad producto importaciones de las manufacturas industriales. 


\section{CONCLUSIONES}

A lo largo del trabajo, y dentro de los límites previstos por la extensión del artículo, se buscó presentar una síntesis del posicionamiento de Argentina en los distintos PTE. En este sentido, la sucesión de los modelos de desarrollo adoptados a nivel interno y los cambios tecnológicos impulsados por cada paradigma global expresan la reconstitución de las fuerzas productivas domésticas e internacionales sin advertir un cambio estructural en el posicionamiento periférico de Argentina en la economía internacional.

Si bien no existe un proceso lineal para dicha reproducción periférica en la relación entre sistema productivo, capacidades tecnológicas e inserción externa, es posible identificar hechos estilizados de cierta regularidad tanto de carácter doméstico como internacional. Entre los factores externos se deben señalar los cambios en los precios relativos; las diferentes coyunturas sistémicas; las condicione asimétricas de movilidad y transferencia de tecnología; y los movimientos de tecnologías declinantes junto con capitales financieros hacia la periferia en momentos de crisis en el centro.

Por su parte, en el plano interno -y de forma más específica- pueden señalarse algunos elementos de significativa relevancia analítica. En primer lugar, las bondades de la pampa húmeda como centro de la estructura productiva local que logró posicionar a la Argentina entre las principales economías del mundo sin desarrollar capacidades tecnológicas. En segundo lugar, la vulnerabilidad frente a los acontecimientos externos de un modelo dependiente derivó en la estrategia sustitutiva y la generación obsolescente de capacidades tecnológicas como respuesta tardía a un PTE en declinación, bajo una dinámica de formación exógena y transnacionalizada.

En tercer lugar, hacia la década de 1970 se produjo la consolidación de la heterogeneidad productiva, con un acercamiento del sector primario a la frontera tecnológica pero sin generar capacidades innovativas locales. Mientras tanto el sector industrial logró una débil sobrevida sobre una estructura concentrada y de enclaves, con escaso desarrollo tecnológico y un sistema de innovación que desde lo institucional comenzó a formalizarse pero alejado de la base productiva y una visón de autonomía. En los últimos años, a partir de la reactivación productiva luego de la crisis del orden convertibilidad, se evidenció un fortalecimiento organizacional del sistema de innovación pero sin una ruptura estructural en el campo productivo y el posicionamiento periférico. 
Por último, estos elementos y la caracterización del paradigma actual en la identificación de los sectores en crecimiento hacia la formación de un nuevo PTE, abren camino hacia la elaboración de modelos analíticos desde una perspectiva contextualizada y dinámica, conscientes de los elementos en transformación a nivel sistémico y de los límites de las estrategias de desarrollo implementadas localmente.

\section{REFERENCIAS BIBLIOGRÁFICAS}

Albornoz, M. \& Gordon, A. (2011). La política deficiencia y tecnología en Argentina desde la recuperación de la democracia (1983-2009). En M. Albornoz. M. \& J. Sebastián (Eds.), Trayectorias de las políticas cientificas y universitarias de Argentina y España (pp.1-46). Madrid: CSIC.

Bekerman, M. (2015). Restricción externa al crecimiento de Argentina. El rol de las manufacturas industriales. Problemas del desarrollo, 183 (46), 59-88.

Bresser-Pereira, L \& Gala, P. (2008). ¿Por qué el ahorro externo no promueve el crecimiento?. Investigación económica, 67(263), 107-130.

Curia, E. L. (2011). El modelo de desarrollo en la Argentina. Los riesgos de una dinámica pendular. Buenos Aires: Fondo de Cultura Económica.

De Angelis, I. (2015). Cambio tecnológico en el modelo de desarrollo posconvertibilidad. Límites y oportunidades para la transformación de la estructura productiva. Un análisis de la industria de bienes de capital en Argentina. (Tesis de Maestría sin publicar). Universidad Nacional de Quilmes, Bernal.

Féliz, M. (2011). ¿Neo-desarrollismo: más allá del neo-liberalismo? Desarrollo y crisis capitalista en Argentina desde los 90. Revista THEOMAI (23), 72-86

Freeman, C. \& Pérez, C. (2003). Crisis estructurales de ajuste, ciclos económicos y comportamiento de la inversión. En F. Chesnais \& J. Neffa (Comp.). Ciencia, tecnología y crecimiento económico (pp. 211-243). Buenos Aires: CEIL-PIETTE/CONICET.

Jaguaribe, H. (1975). Por qué no se ha desarrollado la ciencia en américa latina. En J. Sábato. El pensamiento latinoamericano en la problemática ciencia-tecnología-desarrollo-dependencia (pp. 95-115). Buenos Aires: Editorial Paidós.

Katz, J. (2000). Reformas estructurales, productividad y conducta tecnológica en América Latina. Santiago de Chile: Fondo de Cultura Económica, CEPAL.

Llach, J. (1984). El plan Pinedo de 1940, su significado histórico y los orígenes de la economía política del peronismo. Desarrollo Económico 23(92), 515558.

López, A. (2002). Industrialización sustitutiva de importaciones y sistema nacional e innovación: un análisis del caso argentino. Redes, 10(19), 43-85. 
Nochteff, H. (2002). ¿Existe una política de ciencia y tecnología en la Argentina? Un enfoque desde la economía política. Desarrollo Económico, 41(164), 555-578.

Nun, J. (1995). Argentina: el Estado y las actividades científicas y tecnológicas. Redes, 2(3), 59-98.

O’Donnell, G. (1977). Estado y alianzas en la Argentina, 1956-1976. Desarrollo Económico, 16(64), 523-554.

Pérez, C. (2004). Revoluciones tecnológicas y capital financiero. La dinámica de las grandes burbujas financieras y las épocas de bonanza. México: Siglo XXI.

Pérez, C. (2009). La otra globalización: los retos del colapso financiero. Problemas del Desarrollo. Revista Latinoamericana de Economía, 40(157), 11-37.

Porta, F. \& Bianco, C. (2004). Las visiones sobre el desarrollo argentino: consensos y disensos. Centro REDES, Documento de Trabajo No. 13.

Rapoport, M. (2000). Historia económica, política y social de la Argentina (18802000). Buenos Aires: Macchi.

Sábato, J. \& Botana, N. (1970). La Ciencia y tecnología en el desarrollo de América Latina. En A. Herrera (Ed.), América Latina: ciencia y tecnología en el desarrollo de la sociedad (pp.59-76).Santiago de Chile: Editorial Universitaria.

Schvarzer, J. (1996). La industria que supimos conseguir. Buenos Aires: Editorial Planeta.

Vitelli, G. (2012). Los dos siglos de la Argentina. Historia Económica Comparada. Buenos Aires: Centro Cultural de la Cooperación.

(C) 2016 por los autores; licencia otorgada a la Revista Estudios Económicos. Este artículo es de acceso abierto y distribuido bajo los términos y condiciones de una licencia Atribución-No Comercial 3.0 Unported (CC BY-NC 3.0) de Creative Commons. Para ver una copia de esta licencia, visite http:// creativecommons.org/licenses/by-nc/3.0/ 
